\title{
¿HACIA DÓNDE VA LA ENSEÑNANZA DE LA HISTORIA Y DE LAS CIENCIAS SOCIALES? APUNTES PARA LA COMPRENSIÓN DE UN DEBATE
}

\author{
Joan PAGĖS BLANCH
}

Profesor de Didactica de las Ciencias Sociales. Universitat Autònoma de Barcelona

Resumen: La enseñanza de la historia y de las ciencias sociales ha sido objeto de largas $y$ estériles polémicas durante el siglo $X X$ que se han centrado fundamentalmente en la selección de los contenidos escolares y sus propósitos educativos. En las dos últimas décadas del siglo XX se han vivido nuevos debates en todo el mundo que, en general, culminan con una reforma del curriculo prescrito que suele tener poco impacto en la práctica.

En este trabajo se presenta una panorámica general de la naturaleza de estos debates ejemplificada, en primer lugar, en un análisis critico del Informe de la Real Academia de la Historia sobre la enseñanza de la misma. También se presentan distintos enfoques sobre la procedencia y la naturaleza de los conocimientos sociales escolares y algunos resultados de investigaciones centradas en el aprendizaje. El trabajo concluye con algunas propuestas para el futuro.

Durante buena parte de los ochenta y de los noventa la enseñanza de la historia y de las ciencias sociales fue motivo de agrias polémicas en todo el mundo occidental, como, por otro lado, lo habia sido durante todo el siglo XX y lo sigue siendo hoy. En realidad, los debates actuales no son sino una continuación de otros debates anteriores. ¿Por qué el curriculo y la enseñanza de la historia y de las ciencias sociales están continuamente en el centro de las polémicas educativas?, ¿Qué ocurre en las aulas cuando se enseñan y se aprenden ciencias sociales, historia, geografia,...?

Mi proposito en este articulo es presentar algunas de las razones que explican el debate asi como caracterizar las posiciones en torno al mismo. Se trata de un debate de naturaleza fundamentalmente ideológica que suele tener poca relación con lo

ENDOXA: Series Filosoficas, n. 14, 2001, pp. 261-288. UNED, Madrid 
que ocurre en las escuelas cuando se enseñan y se aprenden ciencias sociales, geografia o historia. Es un debate universal que se centra fundamentalmente en los contenidos escolares que deben formar parte del curriculo y en sus propositos.

El trabajo consta de cinco apartados. En primer lugar, presento una lectura crítica del Informe de la Real Academia de la Historia, Informe que, como es sabido, avivo el debate a finales del pasado curso académico. El segundo apartado lo dedico al análisis de la procedencia y la naturaleza de los contenidos historicos y sociales escolares. En el tercero presento los dos enfoques que han polarizado el debate hasta la actualidad: el enfoque mayoritario centrado en los contenidos disciplinares y el enfoque minoritario centrado en los problemas sociales relevantes, y planted una via alternativa - la educación para la formación de una ciudadania democrática - que recoge aportaciones de ambos enfoques. El cuarto apartado contiene algunas apreciaciones sobre los resultados de investigaciones sobre el aprendizaje del saber social e historico escolar que sirven de contrapunto a los debates sobre la naturaleza de los contenidos sociales escolares y el logro de sus propositos. Finalmente, concluyb con una breves consideraciones sobre la necesidad de potenciar más la investigación didáctica, sobre la necesidad de repensar la formación del profesorado y sobre la necesidad de afrontar los retos que la enseñanza de la historia y de las ciencias sociales presenta hoy con una clara vision de futuro.

Una lectura crítica del Informe de la Real Academia sobre la enseñanza de la historia ${ }^{3}$

Cualquiera que recuerde el debate suscitado hace ya casi tres años en relación con la enseñanza de la historia y de las humanidades, podrá comprobar que el Informe de la Real Academia de la Historia no solamente no ha aportado nada nuevo y de utilidad para la enseńanza y el aprendizaje de la historia sino que, debido a su eco en los medios de comunicación, ha seguido confundiendo a la opinión pública. Tres años después de la conferencia de Esperanza Aguirre en la sede de la Real Academia de la Historia estamos donde estábamos, por lo que siguen siendo válidas la mayoría de las

1 Parte de este trabajo se ha publicado con el mismo título en Cuadernos Cives de Información y documentación n. 12 , octubre 2000, 13-18. 
críticas y de las sugerencias que realizamos entonces (por ejemplo, Pagès, 1998a, 1998b, 1999).

¿Qué ocurre con la enseñanza de la historia ahora y aque? Como señala el Informe, bastante acertadamente, no se sabe: "faltan noticias sobre un elemento esencial: la actividad docente desarrollada por cada profesor en su aula». Ciertamente se sabe poco aún de lo que ocurre en las aulas cuando se enseña y se aprende historia, pero se sabe más de lo que creen los académicos que han redactado el Informe y la mayor parte de quienes han intervenido en el pasado debate. En los últimos años se ha desarrollado una importante investigación en didáctica de la historia y de las ciencias sociales que ha puesto de relieve muchas más cosas de las que al parecer conocen los expertos de la Academia y las propias autoridades ministeriales quienes, por otro lado, suelen ser bastante poco generosas a la hora de dotar de recursos a la investigación en didáctica de la historia y de las ciencias sociales. Son varias las Universidades que a través de sus departamentos de Didáctica de las Ciencias Sociales han impulsado programas de doctorado en Didáctica de las Ciencias Sociales y Didáctica de la Historia y líneas de investigación para intentar averiguar qué ocurre en las aulas y buscar alternativas a sus problemas. También cabe recordar el enorme crecimiento producido en los últimos años de las publicaciones dedicadas a la enseñanza y el aprendizaje de las Ciencias Sociales y de la Historia.

Sin embargo, y a pesar de este aún escaso conocimiento de lo que ocurre en las aulas, en mi opinión, se puede afirmar sin temor a equivocarse demasiado que con la enseñanza de la historia ocurre ni más ni menos lo mismo que ocurre con otras disciplinas escolares. Hay alumnos a quienes les gusta la historia y la aprenden, y otros alumnos a quienes no les gusta y no la aprenden. $\mathrm{Y}$ otros a quienes no les gusta especialmente pero tienen éxito -apruebanen su estudio. Como ocurre con las otras asignaturas y como ha ocurrido casi siempre. Por otro lado, hay profesores que saben historia y además saben como hay que enseñarla y otros profesores que saben historia pero no saben como enseñarla. $Y$, tal vez, profesores que no saben ni una cosa ni la otra ni les interesa aprenderlas. Como en el resto de asignaturas y en el resto de profesiones. Como en la vida misma.

¿Cuál es, entonces, la razón del Informe de la Real Academia?, ¿qué aportaciones se hacen al conocimiento de la enseñanza y el aprendizaje de la historia escolar?. Intentaré dar respuesta a estas preguntas desvelando algunas de las 
principales incoherencias y medias verdades, cuando no falsedades, del Informe.

La primera ya la he seńalado: se pretende hacer un diagnóstico de una realidad que se desconoce. A partir de esta constatación, que no aparece hasta casi el final, el Informe cae por su propio peso. Ya no es un problema de rigor cientifico, es un problema de ética. Al desconocerse lo que ocurre en la práctica se opta por identificarla con el currículo y con los libros de texto.

En relación con el currículo - concepto que no parece del agrado de los académicos que han redactado el Informe-, los autores parten de un supuesto que la investigación educativa internacional hace tiempo que se ha encargado de desmentir. Creen que cambiando los contenidos, substituyendo las historias locales y regionales, por una "historia común" cambiaran las prácticas.

El cambio del currículo escolar o de los contenidos de las disciplinas escolares, no ha supuesto nunca el cambio de las prácticas de enseñanza. Ninguna práctica social - y la enseńanza de la historia y de las ciencias sociales como la de cualquier otra asignatura, es una práctica social - cambia por la voluntad del legislador, cambia cuando los prácticos quieren cambiarla. Desde luego, un cambio del currículo bien hecho, con medios y formación, puede facilitar al profesorado la posibilidad de contrastar su práctica con las propuestas que se realizan desde la administración y puede promover el cambio. Por el contrario, un cambio sin medios ni formación suficiente facilita la continuidad y garantiza la pervivencia de las prácticas que se pretenden modificar.

El currículo de la LOGSE - y ésto parece que lo ignoran los autores del Informe- apostó por un modelo radicalmente diferente al existente anteriormente. Frente a una concepción muy cerrada y prescriptiva de los planes de estudio en la que se detallaban todos y cada uno de los contenidos, curso por curso, la LOGSE apostó por un modelo abierto, y poco prescriptivo en relación con la selección y secuencia de los contenidos. Apostó por un currículo democrático que dejaba a los centros y a los profesores los aspectos más importantes de la enseñanza: concretar aquello que deben y pueden aprender alumnos y alumnas con nombres y apellidos y ordenarlo en itinerarios que, ante todo, consigan buenos aprendizajes. Potencialmente es un currículo altamente profesionalizador pues deja al profesorado la posibilidad de desarrollar sus competencias sin necesidad de ser tutorizados en la toma de sus decisiones por historiadores, políticos, pedagogos o cualquier otro tipo de expertos. Por primera vez en la historia de España, el currículo escolar se democratizó y su cons- 
trucción dejó de ser patrimonio exclusivo de unos supuestos expertos (historiadores, pedagogos, etc.). ¿No será que los miembros de la Real Academia de la Historia quieren recuperar el protagonismo que tenían antaño en la prescripción de aquello que de historia debían enseñar los profesores y aprender sus alumnos?, ¿por qué no intervinieron en el debate que existió en España a lo largo de la década de los 80 cuando se estaba experimentando la reforma curricular y esperaron al triunfo electoral de los conservadores para hacer oír su voz?

También ignoran los autores del Informe que un currículo abierto al ser poco prescriptivo deja un amplio margen de maniobra a quienes son los responsables de concretarlo: los profesores, a quienes, después de cursar estudios universitarios, se les debería considerar suficientemente preparados para tomar este tipo de decisiones.

Por otro lado, no es de rigor atribuir parte de los males de la enseñanza de la historia al hecho de que ésta forme parte de un área de conocimientos, el área social. El área de conocimiento es un territorio curricular en el que se incluyen disciplinas cuyo objeto de estudio tiene una cierta afinidad. En ningún caso este hecho ha supuesto ni supone la desaparición de la historia o de cualquier otra disciplina clásica, como puede comprobarse, por ejemplo, en los países con más tradición en la organización curricular por áreas (Estados Unidos, Canadá, etc...). Como he defendido en alguna otra ocasión (Pagès 1991), en el currículo de la ESO puede haber tanta historia y tanta geografia como se quiera. Otro tema es la conveniencia de que exista más historia contemporánea que historia de otros períodos anteriores. Tal vez el legislador pensó que se tenía que evitar lo que ocurría habitualmente antes de la LOGSE: casi nunca se estudiaban los tiempos más recientes, es decir no "había tiempo" para llegar al siglo XIX y, en especial, al XX.

Finalmente tampoco es muy científico cargar contra el currículo de las Autonomías, en especial el de las Autonomías dirigidas por partidos nacionalistas, utilizando dos casos que tienen escasa relación con lo que se ha hecho o dejado de hacer en Catalunya y el País Vasco, por ejemplo. Como se sabe , el currículo de Andalucia ha sido elaborado y aprobado por una administración socialista, mientras que el currículo gallego lo ha sido por un gobierno del Partido Popular. No seré yo quien defienda la utilización partidista del currículo de historia por parte de los nacionalistas o de los regionalistas tanto de los denominados "periféricos" como de los centralistas. Sorprende, sin embargo, que los académicos autores del Informe se alineen con las tesis españolistas del 
Partido Popular, y le den cobertura "científica", cuando tampoco se ha demostrado nunca que la enseñanza de la historia sirva para crear en nuestros alumnos una conciencia nacionalista.

A la vista de tan escasos argumentos, los autores del Informe vuelven a confundir a la opinión, identificando la enseñanza de la historia con los libros de texto. Ciertamente, en los libros de texto hay historia pero no hay enseñanza ni aprendizaje. Los libros de texto no son la enseñanza de la historia, son un medio que puede ser utilizado para enseñar y aprender historia. ¿Qué se sabe sobre su uso en las aulas? Nada o muy poca cosa. Lo cómodo es analizar sus contenidos. Y de nuevo, los autores del Informe vuelven a las extrapolaciones y a las generalizaciones fáciles y altamente demagogas a partir del análisis de los libros de texto de dos editoriales. Desde luego, si aplican los mismos métodos a la investigación histórica, apañada está la historia!

Está bien que a los autores del Informe les preocupe la historia. Debería haberles preocupado también su enseñanza y, en especial, su aprendizaje. Parece que se olvidan que si se enseña historia es para que se aprenda historia. Las reglas de la enseñanza y del aprendizaje de una disciplina, en nuestro caso la historia y el resto de disciplinas sociales, no son las reglas de la investigación histórica ni tiene más relación con ella que la que se deriva de la pertenencia de los saberes a los distintos paradigmas o escuelas de pensamiento existentes y por supuesto a su coherencia científica. Los saberes escolares no son los saberes científicos, aunque han de ser científicos, pues ni tienen las mismas finalidades ni se producen en los mismos contextos de creación y divulgación, sin embargo, no pueden ser saberes obsoletos ni saberes adulterados. Son saberes que para cumplir sus objetivos necesitan ser adaptados y convertidos en saberes escolares.

La enorme cantidad de saberes del conjunto de ciencias sociales y, en particular de la historia, ha conducido a algunos expertos curriculares a buscar criterios para una adecuada selección y secuencia que evita repeticiones innecesarias y permita sentar las bases para seguir aprendiendo. Frente a la enorme cantidad de saberes factuales y de "grandes personajes" que se han ido repitiendo una y otra vez a lo largo de la escolaridad, existen propuestas que se basan en criterios más cualitativos que la simple selección y acumulación de aquellos elementos - "grandes personajes y acontecimientos políticos"-, que, según el Informe, constituyen, junto con la cronología «las señas de identidad» y «el armazón" de la disciplina. 
Aprender historia es sin duda conocer hechos y situaciones del pasado. Pero también es saber que estos hechos y estas situaciones no son el pasado sino una interpretación de lo que sucedió realizada por los historiadores a partir de unos determinados métodos y desde unos determinados supuestos ideológicos. ¿No se puede enseñar, no se puede aprender cómo se construye la historia ni por qué se construye como se construye? De nuevo se recurre al tópico fácil —en el currículo se da se da más importancia al cómo enseñar que al qué enseñar; los hechos y los grandes personajes han sido sustituidos por los procedimientos y por las actitudes. ¿¿Cuándo ha sucedido ésto?, ¿dónde?, ¿en los libros de texto?, jen las aulas?. No se sabe.

El Informe cae en el absurdo de desprestigiar la pedagogía, como si la actividad docente no tuviera necesidad de la misma. Enseñar es algo más que saber historia y saber contar historias, es conseguir que el alumnado aprenda historia. Provocan hilaridad afirmaciones como que "los académicos (...) saben de la ignorancia —cuando no de la tergiversación - de la historia que padecen los alumnos", $y$ "sufren de la imposibilidad de formar a sus alumnos». ¿Se han preguntado las razones de esta supuesta ignorancia?, ¿saben quiénes han formado a los profesores y profesoras que enseñan historia, para qué los han formado y cómo los han formado?, ¿qué diríamos si estas palabras las hubiesen pronunciado otros profesionales en relación con sus usuarios, por ejemplo los médicos o los arquitectos? Sorprende, y causa una cierta vergüenza ajena, leer que uno de los problemas de la enseñanza de la historia es ula obsesión pedagógica" y sus efectos negativos al "poner tanto énfasis en los métodos de la enseñanzaw. Revísense los planes de estudio de las carreras de historia o de oras humanidades de las Universidades españolas y señálese donde está la pedagogía o la didáctica —expresión, por cierto, que al parecer desconocen los miembros de la Real Academia que han redactado este Informe pues sólo la utilizan una vez y no para señalar las aportaciones que ha hecho en relación con la formación del profesorado para enseñar historia y con la investigación sobre la enseñanza y el aprendizaje de la misma- que justifica hablar de "obsesión". Hoy por hoy, toda la pedagogía que aprenden quienes se preparan para ensenar historia y la que aprendieron quienes ya la enseñan se reduce a un cursillo de un trimestre de duración, el CAP, y en poquísimas ocasiones a una didáctica de la historia que figura como asignatura optativa en algunos planes de estudio de las carreras de historia.

En fín... esperemos que el Informe de la Real Academia de la Historia no pase a la historia como un ejemplo de lo que los historiadores pueden aportar 
a la enseñanza de la historia. Esperemos que se pueda reconducir el debate y sacarlo del campo de lo político y de los medios. No porque los debates educativos, y en especial éste, no sean debates políticos ni porque la sociedad no deba estar informada y tener opinión sobre lo que se hace o se deja de hacer en las escuelas y en los institutos, sino por la necesidad de que sea el profesorado y los expertos en educación, en didáctica, en psicología, en pedagogía quienes, junto sin duda con los historiadores y otros científicos sociales, analicen los problemas reales de la enseñanza y del aprendizaje de la historia y del conjunto de ciencias sociales y humanas. El debate sobre la instrumentalización política de la historia por parte de los nacionalismos, de todos los nacionalismos incluido el español, difícilmente irá más allá de donde está ahora y desde luego no resolverá los problemas reales de la enseñanza y del aprendizaje de la historia. Al contrario, de seguir como ha seguido hasta ahora cada vez confundirá más a la opinión pública como ha ocurrido en los Estados Unidos (véase el interesante trabajo de Johnson y Avery, 1999, sobre como la prensa norteamericana analizó el currículo de historia para los centros públicos de este país y aplíquese su metodología al análisis de los artículos de opinión publicados en la prensa de nuestro país), que puede acabar no sólo desinteresándose del tema sino apoyando aquellas medidas que acaben de raíz con el problema suprimiendo, por ejemplo, una enseñanza que por un lado se les presenta como muy problemática pero que, por el otro, suele considerar poco útil. Además puede profundizar el abismo entre quienes pretenden resolver los problemas de los prácticos y los propios prácticos de quienes depende, no se olvide, lo que ocurra o deje de ocurrir en las aulas.

La procedencia y la naturaleza de los contenidos históricos y sociales escolares

Este debate, y el que le precedió en tiempos de Dona Esperanza Aguirre, es un ejemplo de la compleja tarea de dilucidar qué contenidos sociales e históricos, y para qué, deben formar parte del contenido escolar. Debates parecidos se han sucedido y se suceden en otros países de nuestro entorno. Suelen ocupar páginas y más páginas de los medios de comunicación y, por supuesto, son también objeto de preocupación de los expertos en temas curriculares, de los didactas y de buena parte del profesorado. A diferencia de lo que ocurre en otras áreas de conocimiento y en otras disciplinas escolares, donde el debate 
también es inevitable porque es imposible enseñarlo todo y se requieren criterios para seleccionar lo más relevante y secuenciarlo a lo largo de las distintas etapas educativas y de los cursos, en el caso de las Ciencias Sociales y de la Historia, el debate se politiza frecuentemente y adquiere un carácter tan ideológico que a menudo se olvida que se discute sobre contenidos escolares, es decir, sobre aquello que potencialmente puede ser más educativo para las futuras generaciones.

Goodson (1995) señala que «la escuela siempre ha sido un "terreno de enfrentamiento" donde las fuerzas e influencias de diversos grupos sociales han luchado para conseguir que se diera prioridad a sus propósitos" de tal manera que "el curriculum escolar puede verse como portador y distribuidor de prioridades sociales» (53). Para este autor, el currículum escolar es "un artefacto social, concebido y hecho para propósitos humanos deliberados" (95), es una construcción social, cambiante y sometida a todo tipo de presiones por lo que no puede considerarse desde una perspectiva neutral.

Si esto es así para el conjunto de disciplinas escolares - el propio Goodson lo ha estudiado para el caso de las matemáticas, de las ciencias y de la lenguatambién lo es, y quizás con más motivo, para el caso de aquellas disciplinas escolares cuyo objeto de enseñanza es la propia sociedad y sus protagonistas, las relaciones entre ellos y con las demás sociedades en el presente, en el pasado y aún en el futuro.

Los problemas de la selección de los contenidos sociales escolares arrancan del hecho de que son contenidos sobre la realidad social y ésta, como es sabido, es una realidad compleja, en continua construcción, e interpretable en función de los distintos intereses económicos, sociales, políticos y culturales existentes. No es posible pensar la realidad social, objeto de estudio escolar, en términos de una supuesta ideología de la neutralidad. Nunca ha sido así ni nunca lo será. Es posible, sin duda, consensuar democráticamente unos contenidos, o unos criterios para su selección, pero en una sociedad democrática hay que respetar el pluralismo ideológico, social, cultural y epistemológico también en el ámbito curricular y escolar.

No existe suficiente investigación sobre los contenidos sociales potencialmente más educativos, por lo que el debate sobre su selección no cuenta con el soporte de unos resultados contrastados empíricamente. Por esta razón, los argumentos que suelen utilizarse en función de las posiciones en debate, adolecen de la credibilidad que tienen en otros ámbitos escolares. 
Aparentemente, el debate sobre la selección de los contenidos sociales escolares tiene hoy como protagonistas a los partidarios de un currículo escolar centrado en las disciplinas, más concretamente, a los partidarios de un currículo de historia y de geografía, por un lado, y a los partidarios de un currículo centrado en los problemas sociales relevantes, por el otro. En el fondo, sin embargo, los protagonistas tienen diferentes concepciones sobre la realidad y el orden social, sobre la identidad nacional y la cultura, o la historia, común, sobre la socialización en unos determinados valores y el concepto de ciudadanía, sobre el protagonismo de las minorías étnicas y de los géneros, o sobre la globalización y sus relaciones con las identidades locales, etc. A alguno de estos aspectos se refiere Hargreaves (1998) cuando señala:

"Además de la regeneración económica, en muchos países se prevé que los profesores contribuyan a reconstruir las culturas e identidades nacionales. La integración económica global, tal como se expresa en la creciente unión económica en Europa y en el Tratado de Libre Comercio en Norteamérica, está sembrando temores de desintegración nacional en países como Gran Bretaña y Canadá - temores de que perderán sus identidades y características específicas culturales y políticas. En consecuencia, como respuesta a la globalización económica y a la migración cultural, en muchos lugares del mundo se prevé que las escuelas carguen con gran parte del peso de la reconstrucción nacional. Por ejemplo, en un esfuerzo para resucitar los valores y el sentido de certeza moral tradicionales, los curricula escolares rellenan con nuevos contenidos que resaltan la unidad e identidad históricas, geográficas y culturales, contenidos que los profesores deben dominar e impartir» (31-32).

Esto es lo que parece que quieren hacer los conservadores españoles con el visto bueno de la Real Academia de la Historia. Pero no sólo para dar respuesta a la globalización económica sino también para frenar las competencias de las Comunidades Autonómicas y la consiguiente descentralización curricular. Cuando por primera vez en la historia de la educación española se opta por un currículo abierto, democrático y descentralizado, con importantes competencias en manos del profesorado, estalla el debate en relación precisamente con la enseñanza de la historia de España y la supuesta cultura común. Parece que frente a los intentos de construir en el medio plazo un currículo europeo, con una historia de los europeos y de las europeas que supere los clásicos enfrentamientos entre paises, $y$ ante el hecho indiscutible de una diversidad cultural entre los españoles y españolas, se quiere optar por volver a un currículo centralizado, y centralista, que defienda las supuestas esencias de una hispanidad 
entendida como un todo homogéneo. Poco importan los contenidos que debe aprender el alumnado. Lo que importa es garantizar el control social del currículo con la idea de imponer una determinada visión de quienes son buenos ciudadanos y ciudadanas españoles conforme a un modelo construido de un pasado y de un presente sin fisuras ni conflictos.

El currículo de estudios sociales se convierte así, tanto en España como en la mayor parte de países occidentales, en un campo de batalla ideológico y moral (Wayne Ross, 2000). Lo importante no es qué deben aprender los alumnos y las alumnas sobre el pasado y el presente para la construcción de su futuro personal y social, y cómo deben aprenderlo, sino qué imagen de país, de ciudadanía y de participación social debe imponerse para que sigan siendo súbditos sumisos a la voluntad del poder político dominante. Los contendientes en este campo de batalla no siempre tienen conciencia de su papel y a menudo actúan sin saber cuáles van a ser las consecuencias que sus acciones, o sus discursos, tendrán para la enseñanza y el aprendizaje de la historia y de las ciencias sociales.

Es preocupante constatar que en los países donde se ha producido este debate, buena parte de la ciudadanía ni lo ha seguido ni se ha interesado por sus resultados. A menudo se ha conseguido el efecto contrario al perseguido, que se supone que era intentar mejorar el aprendizaje de la historia y de las ciencias sociales en las jóvenes generaciones. Cuando hace ya unos años, la Sra. Tatcher optó por cerrar el curriculum de historia e imponer un modelo homogéneo a todos los centros ingleses y galeses, algunas asociaciones de profesores de historia buscaron el apoyo de los padres de los alumnos y alumnas a fin de poder seguir desarrollando sus competencias curriculares. Esta fue su respuesta en palabras de Arkell (1986):

"This is because many adults have happily forgotten almost all the history that they learned at school or else have vivid memories of the arid and irrelevant details that were inlicted on them there and can see little point in subjecting their own children to anything similar. Even those with positive memories of their own history teaching known that history will rarely help a child secure employment. And so the case for retaining, or even expanding, history in the school curriculum must be made on other grounds; in particular its potential role as a socializing agent» (Arkell, 1986, 29-30).

Los propuestas de los políticos conservadores ingleses y norteamericanos, o de algunos políticos del centroizquierda europeos, en relación con su modelo 
de enseñanza de la historia y de las ciencias sociales, han tenido más éxito a causa de las rutinas existentes en esta enseñanza que no como consecuencia de su valor educativo real. Si bien es cierto que el debate no es un debate que atraiga a la mayoría de la ciudadanía, también lo es que esta misma mayoría no se opone a que sus jóvenes reciban una formación histórica y geográfica parecida a la que recibió ella misma en su época estudiantil aunque se hayan producido cambios en el mundo $y$, probablemente, las jóvenes generaciones necesiten otra formación y otros contenidos para poder ubicarse en su mundo y participar en él.

Conocimientos disciplinares, problemas sociales relevantes y educación para una ciudadanía democrática

El problema de los contenidos sociales escolares deriva del hecho de que el objeto de estudio de esta área de conocimiento es el más inclusivo de todas las áreas de conocimiento escolares. Sin embargo, el problema de la selección de los contenidos no consiste sólo en decidir qué parte del conocimiento de la historia y de las otras ciencias sociales se traspasa a la escuela, sino que consiste en prever el carácter dinámico e inclusive del currículo como un instrumento que, simultáneamente, ha de contemplar a los estudiantes, a los profesores, a las disciplinas y al contexto. Para decidir los límites de lo que conviene enseñar en las escuelas, de lo que es más conveniente que aprendan las jóvenes generaciones, Wayne Ross (1997) cree que es necesario tomar decisiones sobre los siguientes aspectos: cuál es el conocimiento social más importante, cuáles son las habilidades y las conductas más valiosas, qué valores son más significativos y qué secuencia de contenidos y habilidades es la más adecuada para los conocimientos y los estudiantes. Esta toma de decisiones ha generado a lo largo del siglo XX debates intelectuales sobre los propósitos, el contenido y la didáctica de los estudios sociales, y los sigue generando.

Los estudios sociales, en su sentido más amplio, pretenden preparar a las jóvenes generaciones para que tengan los conocimientos, las habilidades y los valores necesarios para participar activamente en la sociedad. En sus orígenes, se podían considerar un elemento fundamental de la educación nacionalista, pues se trataba fundamentalmente de preparar patriotas leales. A lo largo del siglo XX han aparecido otras opciones, otros propósitos, sin embargo no ha desaparecido el enfoque nacionalista de estos estudios, entre otras razones por- 
que no existe consenso sobre el significado del concepto de ciudadanía, y de sus implicaciones educativas, concepto del que se reclaman deudores los partidarios de los distintos enfoques existentes en la actualidad.

Para unos, la ciudadanía supone el aprendizaje de una cultura común, es decir la adaptación a las normas sociales dominantes, la socialización. Para otros, lo importante para una buena ciudadanía es el conocimiento disciplinar en sí mismo, considerado no como un medio sino como un fin. Finalmente, para otros, la ciudadanía se alcanza cuando los conocimientos sociales se consideran un medio para la promoción y el desarrollo del pensamiento crítico y reflexivo, es decir son una herramienta para la participación y la transformación social. Los dos primeros enfoques se consideran cercanos a las posturas políticas conservadoras, mientras que el tercer se identifica con la tradición educativa progresista. Esta clasificación no es, en absoluto, una clasificación cerrada. Al contrario, dentro de cada enfoque conviven posiciones diferentes y aún contradictorias entre sí. Por ejemplo, dentro del enfoque centrado en las disciplinas, coexisten partidarios de una enseñanza basada en la simple transmisión cultural de una realidad social homogénea, y partidarios de una enseñanza basada en el desarrollo del pensamiento crítico y en una sociedad plural y diversa. En ambos casos, comparten sin embargo, su interés para conseguir sus fines a través del conocimiento disciplinar (Stanley/Nelson, 1994; Wayne Ross, 1997).

El predominio de un enfoque o de otro está, en buena parte, condicionado por las políticas educativas y curriculares existentes en cada momento histórico. Y por las influencias que en ellas han tenido distintos sectores sociales, desde las asociaciones profesionales hasta las editoriales de libros de texto. En este sentido, el análisis de la evolución del currículo español en los últimos cincuenta años puede ilustrar a la perfección las diferencias entre los distintos enfoques. Al currículo centralista de los tiempos de la dictadura se le opuso un currículo descentralizado en la democracia que recogía gran parte de las aportaciones de los movimientos de renovación pedagógica. Las críticas recientes al currículo de la LOGSE se asientan, no sólo en la victoria política de los conservadores españoles, sino también en el corporativismo de determinados asociaciones profesionales. En determinados momentos del desarrollo del currículo de la LOGSE el peso de las editoriales de libros de texto fue determinante en la concreción no sólo de los contenidos sino del propio proceso de implantación de la reforma. 
En la actualidad, casi nadie discute que los propósitos y las finalidades del currículo, así como sus contenidos y sus objetivos, no pueden identificarse con los documentos curriculares aprobados por las administraciones políticas. El currículo incluye desde estos documentos hasta las prácticas reales en el aula. En consecuencia, parece lógico que se compartan responsabilidades en su construcción y en su desarrollo. Sin embargo, no es ésta la opción que mayoritariamente defienden los gobiernos de la mayor parte de las democracias occidentales. Su opción por un currículo centralizado, por establecer los contenidos que debe aprender toda la población escolar de un país, ha sido considerado por algunos autores como "antidemocratic because they severely restrict the legitimate role of teachers and other educational professionals as well as the public in participating in the conversation about the origin, nature, and ethics of knowledge taught in the social studies curriculum» (Wayne Ross, 1997: 15).

En los USA actualmente el debate está polarizado, como ya he señalado, entre los partidarios de un currículo centrado en el conocimiento disciplinar, fundamentalmente en la enseñanza de la historia y la geografía, y los partidarios de un currículo basado en los problemas sociales relevantes. Los partidarios de ambos puntos de vista creen que los estudios sociales tienen un papel crucial en las concepciones y la comprensión de los roles sociales, políticos y económicos de los ciudadanos y de las acciones que se realizan según sean estas concepciones. Sin embargo, las características de este debate, común por otro lado en muchos otros países, entre ellos España, pueden tener consecuencias mucho más negativas que positivas para la enseñanza de las ciencias sociales, como lo señala Whelan (1997: 21-22) en relación con los mismos Estados Unidos:

"Rather than engaging in a critical yet constructive discussion of their respective positions, prominent spokespersons for both history and issues-centered curriculum models have taken rigid, uncompromising stands, devised historical interpretations to bolster their competing claims of legitimacy, and assailed each other's proposals as inintellectual, antiegalitarian, and a threat to the nation's basic institutions (...).

Furthermore, like many educational policy disputes, this debate is increasingly becoming and end in itself and, as such, of little practical consequence for social studies teachers. It is not that the issues involved are inconsequential, but that they have been confused and obscured by people on both sides 
of the question pressing ever more arcane or irrelevant arguments in an effort to gain some dubious debating advantage. The recent spat (Barth et al. 1991) about the relative merits of history and social studies as academic disciplines, for example, has done little but lend credence to James Leming's (1989) contention of a growing, dysfunctional gulf between social studies theorists and classroom practitioners".

Whelan, sin embargo, se posiciona por un currículo basado en las disciplinas y, en particular, en la enseñanza de la historia. Entiende que un currículo centrado en la historia no ha de contemplar únicamente el pasado sino que los estudiantes han de poder estudiar aspectos del pasado que estén relacionados con los problemas que afectan sus vidas.

Otros, en cambio, creen que lo fundamental es supeditar el conocimiento disciplinar a objetivos sociales más ambiciosos como la formación de la competencia cívica, o los problemas sociales. Para Saxe $(1997,54)$, por ejemplo, el propósito fundamental de los estudios sociales es socializar a las jóvenes generaciones en los principios y las ideas de la democracia liberal norteamericana:

«The key to civic competence is not the acquisition of knowledge from any one of these academic areas alone, nor knowledge from any particular combination of these areas. Rather, social studies for civic competence involves the application and manipulation of knowledge from the traditional academic areas and other contemporany issues of social, political, and economic issues for the expressed purpose of furthering liberal-democratic ideals. Again, it is not the knowledge of these academic areas that is the only goal, but the student's ability to apply the knowledge to affect liberal-democratic goals».

Evans (1997), por su parte, apuesta por los problemas sociales. En su opinión:

"Issues are the proper focus for social studies because they pose real-life problems, raise areas of doubt, motivate reflection, stimulate the need to gain knowledge, and highlight problematic areas of culture. The focus of an issuescenterd approach is on cultural dilemmas and institutional obstacles to social improvement» (pág. 200).

Este debate también ha llegado a España, si bien es aún un debate minoritario. Los problemas de la enseñanza y del aprendizaje de las ciencias sociales y de la historia son universales y, en consecuencia, el debate sobre los criterios para la selección de los contenidos se enmarca en los mismos parámetros que en todo el mundo. En los últimos diez años han aparecido en España distin- 
tos trabajos -más que investigaciones- que nos permiten establecer una cierta clasificación o, como mínimo, sefialar unas ciertas tendencias que, desde luego, comparten, adaptándolos a la realidad española, los mismos principios señalados anteriormente: una tendencia disciplinar, una tendencia que apuesta por los problemas sociales relevantes, y una tendencia que cree posible un acercamiento entre ambos posicionamientos y que cree que en la práctica educativa no son incompatibles. Como es lógico dicha clasificación ha de ser considerada con suma cautela pues las fronteras entre las tres tendencias son bastante sútiles.

Así, por ejemplo, para Prats $(2000,9)$ «Es importante destacar la sustantividad del contenido de la didáctica de las ciencias sociales, que no es otro que el de procesar adecuadamente (en una relación pedagógica) los resultados de las ciencias sociales conformadas académicamente (por ejemplo: el tiempo histórico, el espacio geográfico, las interacciones sociales, las comunicaciones o transacciones económicas, la organización del poder político)».

Esta sustantividad ha de garantizar la pertinencia, y la autonomía, del saber que se enseña en relación con el saber del que emana. Para Prats, las relaciones entre las distintas ciencias sociales escolares pertenecen al ámbito de la metodología de la enseñanza: "La novedad que puede introducir la didáctica de las ciencias sociales (...) estriba en la interconexión propia que el profesor puede establecer entre las metodologías propias de cada disciplina integrada en el área. Ésta es la interconexión que debe ser objeto de formación y el núcleo sobre el que incide la actividad teórica y práctica, así como la determinación de muchos de sus contenidos. Pero, como tal, la interconexión supone respetar la sustantividad de cada una de las ciencias sociales. Sin esta sustantividad no es posible establecer las relaciones especificas que constituyen los contenidos de nuestras acción didáctica, ya que si no fuese asi, estas relaciones tendrian un carácter vacio» (pág. 9). De acuerdo con su propuesta, ulos elementos de la producción del conocimiento de cada una de las disciplinas debe ser un elemento básico en la formación de los docentes" (pág. 15).

Al lado de esta concepción de los contenidos sociales escolares, han ido apareciendo otras propuestas. Tal vez la más consolidada sea la representada por los distintos grupos integrantes del colectivo Fedicaria. En una editorial de su revista conCiencia Social, se afirmaba:

"Siete años de trabajo colectivo que, partiendo de la construcción de materiales curriculares de Ciencias Sociales para la educación secundaria, desde 
supuestos teóricos, científicos y axiológicos comunes -que algunos dieron en llamar con inusual acierto "plataforma de pensamiento"-, nos han conducido a formular las bases de un concepto renovado de la Didáctica Especial de las Ciencias Sociales que no sólo se reclama formalmente heredera de las tradiciones de pensamiento crítico y progresista, sino que integra saberes de muy distinta naturaleza que abarcan desde una reflexión de primer grado acerca de la producción de las disciplinas científicas que informan el currículum escolar, hasta el análisis crítico de los procesos de transferencia cultural que tienen lugar en los contextos escolares, pasando, cómo no, por ensayar mecanismos de selección y organización del currículum, así como diseños de instrucción, que hagan posible modelos $\mathrm{y}$ formas de conocimiento escolar más formativos y críticos con la realidad social en la que se desarrolla la vida del alumnado" (Cuesta, 1999: 9-10).

En el mismo número del que procede la editorial anterior, escribían sobre el tema Gimeno y Cuesta. Para la primera, «existen contenidos de enseñanza que favorecen objetivos de emancipación y pueden ser considerados como potencialmente críticos, como el análisis de conflictos políticos o los que permiten comprender la estructura social y las redes de dominio que la configuran (formas de vida, la sexualidad, el comercio y consumo de drogas, relación entre partidos y sindicatos, relaciones de producción globalizadas y contratación en los países en desarrollo, etc.). Pero existen otros contenidos curriculares de carácter instrumental que están indirectamente referidos a los fines de autonomía y codeterminación y resultan imprescindibles para ello, como el comprender lo que se lee, saber expresar por escrito y oralmente, saber argumentar, manejar el cálculo operatorio o determinados conocimientos del mundo natural . Este tipo de contenidos instrumentales pueden ser críticos o no, según la orientación que se les proporcione" (Gimeno, 1999: 25-26).

Por su parte Cuesta (1999), quien junto con Luis se ha convertido en uno de los pontifices del pensamiento crítico en didáctica de las Ciencias Sociales, apuesta por un currículo centrado en los problemas sociales relevantes sin excluir, para no incurrir en contradicciones con su propia trayectoria profesional e investigadora, la presencia de una cierta visión disciplinar. Expresa sus ideas en los siguientes términos:

«Para empezar la didáctica de la crítica no puede separarse de una problematización del presente (...). Algunos grupos fedicarianos, con mayor o menor entusiasmo, y con distintos matices, tomando como base una plataforma de 
pensamiento crítico, hemos defendido en nuestros proyectos de trabajo una enseñanza orientada hacia el estudio de problemas sociales relevantes del mundo actual. Ciertamente esta posición me parece una condición casi obligada, pero ni mucho menos suficiente de una didáctica de la crítica.

Es obligada porque contribuye a erosionar la ilusión epistemológica que consagra las disciplinas científicas como "formas de conocimiento" reificadas. Reificación consistente en trasmutar hipostáticamente las distintas ciencias sociales en disciplinas escolares en tanto que sustancias intrínsicamente valiosas más allá de los problemas o asuntos que traten y de cómo los traten. (pág. 81).

Otros, como Rozada (1999: 57), por ejemplo, opinan que "el abandono de las disciplinas para organizar los contenidos en torno a una serie de "problemas sociales relevantes" no me parece imprescindible para un enfoque crítico de la enseñanza de las ciencias sociales por dos razones principalmente: a) Porque siguiendo "el hilo" de una disciplina, un profesor que se lo proponga puede abordar de manera recurrente los problemas relevantes de su tiempo y de su contexto. b) Porque los problemas sociales relevantes convertidos en contenido escolar no garantizan por sí mismos el sentido que requiere el buen aprendizaje, pudiendo resultar ser tan académicamente alejados, y por tanto con escaso sentido para los alumnos, como las disciplinas a las que son alternativan.

Las posiciones ejemplificadas por Prats, Fedicaria y Rozada están lejos de los postulados defendidos en España por la Real Academia de la Historia o por el gobierno conservador. Sin duda, pueden considerarse, aún a pesar de no ser coincidentes, como manifestaciones de la creciente preocupación existente en las sociedades democráticas occidentales, y en las democracias emergentes de América Latina y otras partes del mundo, por relacionar el currículo de historia y de ciencias sociales con la formación democrática de la ciudadanía.

En cada país, el debate entre la formación democrática y el currículo de historia y ciencias sociales ha tenido unas características concretas, en función del contexto y de la tradición curricular existente. Sin embargo, es posible señalar unas características comunes que, en opinión de Hursh y Warren Ross (2000) podrían agruparse en tres ámbitos: a) las finalidades y propósitos de la educación y las relaciones entre la educación y la ciudadanía; b) el curriculum de ciencias sociales y el origen y la naturaleza de sus contenidos; y c) las finalidades y la naturaleza del aprendizaje del alumnado. 
En cada uno de estos ámbitos es posible detectar como mínimo dos posiciones que, a grandes rasgos, han sido identificadas como la posición conservadora y la progresista. Para la posición conservadora, las finalidades y propósitos de la educación en general, y de la social en particular, consiste en socializar a las jóvenes generaciones a través del conocimiento disciplinar y utilizando unos métodos que tiendan a su adoctrinamiento. Para los progresistas, - se trata de educar una ciudadanía crítica e informada a través de la incorporación en el currículo escolar no sólo de la perspectiva disciplinar sino de muchas otras perspectivas históricas y culturales emergentes con el fin de aprender a construir sus propios conocimientos y a intervenir en la solución de los problemas sociales.

El problema es que la concepción dominante en Estados Unidos, en la mayoría de países del mundo y también en España, es la posición conservadora. Este es el problema real de la enseñanza y del aprendizaje de las ciencias sociales, de la historia y de la geografia, y la que cuestiona el futuro de tales enseñanzas, porque esta concepción ni cree en la democracia como forma de vida ni cree en el valor que las ciencias sociales pueden tener para formar en los jovenes una profunda conciencia democrática. Wayne Ross (2000), caracteriza el problema y la posible solución con las siguientes palabras:

"The ideology of neutrality that dominates current practices in social studies education (at the elementary and secondary levels, as well as in teacher education and research) is sustained by theories of knowledge and conceptions of democracy that constrain rather than widen civic participation in our society. (...) the theory of knowledge and conceptions of democracy that support what has been called "traditional social studies instruction" function to obscure political-ideological consequences of mainstream social studies. These consequences include conceptions of the learner as passive, democratic citizenship as a spectator project, and, ultimately, the maintenance of status quo inequalities in society" (44).

"What is democratic citizenship? Whitin the standard definition of democracy, citizens should have the opportunity to inform themselves; take part in inquiry, discussion, and policy formation; and advance their ideas through political action. In social studies education, however, democracy is much more narrowly conceived: the citizen is a consumer, an observer, but not a participant" (55).

Las escuelas se convertirán en foros democráticos y la enseñanza de la historia y de las ciencias sociales será un instrumento importante de la formación 
democrática, si son capaces de proporcionar problemas y posibilidades a sus alumnos a fin de hacer emerger la democracia de la diferencia frente a la democracia homogeneizadora que preside el currículo imperante en los Estados Unidos, en España y en casi todo el mundo:

"Multicultural political democracy means that this country was not built by and for only one group, Western Europeans; that our country does not have only one language, English; or only one religion, Christianity; or only one economic philosophy, corporate capitalism. Multicultural democracy means that the keadership within our society sould reflect the richness, colors, and diversity expressed in the lives of all our people. Multicultural democracy demands new types of power sharing and the reallocation of resources necessary to create economic and social development for those who have been systematically excluded and denied" (Kholi, 2000: 36).

\section{El saber social escolar y los aprendizajes}

Los puntos de vista de los historiadores, geógrafos y científicosociales en relación con la selección del contenido social e histórico escolar suelen ser diferentes de los puntos de vista de los didactas y del profesorado. En opinión de Brusa en relación, por ejemplo, con el contenido histórico (1999:67),

"Il ragionamento che fanno gli storici è il seguente: la storia è fatta di queste cose...tra cui ci sono questi aspetti imprescindibili: allora, se vuoi sapere la storia, sono questi che devi imperare. Questo però è un ragionamento giusto, dico io, se vuoi fare lo storico. Invece il ragionamento che fa, o che secondo me dovrebbe fare il docente, è un altro, poiché egli si trova di fronte un ragazzo che non debe fare lo storico, bensì imparare ad utilizzare le conoscenze storiche per arrangiarsi nel mondo contemporaneo, cioè per vivere nel suo mondo. Quindi io insegnante alla storia chiederò certo delle cose vere, profonde, importanti sul piano storico, ma: 1) non ho interesse a chiedere tute le cose importanti della storia; 2) $\mathrm{mi}$ interessa chiedere quelle cose che sono importanti in questo momento".

También son diferentes los puntos de vista de unos y otros de los puntos de vista de los agentes sociales y del alumnado el cual, por regla general, no es considerado a la hora de decidir qué contenidos debe aprender. 
En un interesante trabajo, poco divulgado en España, el periodista francés Jean-Marie Domenach (1989) se preguntaba qué era necesario enseñar en secundaria. Lo hacía de la siguiente manera: «si j'avais entre douze et dixhuit ans aujourd'hui, qu'est-ce que j'aimerais qu'on m'enseigne afin d'accéder le plus vite et le mieux possible à l'essentiel du savoir contemporain?» (p. 9). $\mathrm{Y}$ añadía: «De nos jours, l'accélération du progrès dans les sciences et les techniques, le bouleversement des mentalités et des moeurs sont tels que c'est dans le cours même d'une vie que la transmission des connaissances doit s'accomplir, et presque de soi à soi. La lourde machine de l'instruction publique ne peut évidentment suivre un tel rythme, mais du moins faut-il qu'elle en tienne compte et ne préfere pas, à la reproduction du savoir, sa prope reproduction" (9-10). Se interrogaba sobre si lo que deberían saber los alumnos correspondía a las necesidades de la sociedad y al deber de formar en ellos una personalidad capaz a la vez de satisfacer convenientemente a esta sociedad pero también de comprenderla, criticarla y mejorarla.

Esta preocupación por atender simultáneamente las demandas sociales y los intereses individuales ha estado, y está, en la base de la selección de los contenidos escolares de cualquier área de conocimiento. La sociedad, el alumnado y los contenidos son los tres factores que determinan cualquier programa de estudios. Sin embargo, pocas veces en los debates sobre la selección de los contenidos se ha preguntado a los alumnos de entre doce y dieciocho años que les gustaría aprender como se lo preguntaba Domenach.

Tampoco se han tenido demasiado en cuenta los resultados de las investigaciones sobre los aprendizajes realizados por los alumnos y alumnas durante su escolarización. ¿En qué medida se han alcanzado los objetivos propuestos por los currículos oficiales?, ¿ $S e$ han obtenido aprendizajes cualitativamente distintos desde las propuestas alternativas? Veamos algunos ejemplos de lo que nos dicen investigaciones recientes.

La socialización de los jóvenes y la integración en la sociedad han sido tradicionalmente unas de las principales finalidades de la enseñanza de la historia. Por esta razón constituyó una de los primeros indicadores de la macroencuesta europea "Youth and History" (Angvik/Von Borries, 1977). Kindervater y Von Borries (1997: A 62) escribian al respecto:

"Information about socialization may have great importance for understanding students' historical thinking. The process of socialization is defined 
as the process od developing a personality formed by mutual dependence on the social and material environment in the society (Hurrelmann and Ulrich, 1980, p. 51). The process of socialization may be structurally similar in different countries, even if the content of social learning is very different".

¿Hasta qué punto la ensefianza de la historia y de los estudios sociales ha conseguido y consigue integrar a los jóvenes en su sociedad? Existen ya algunas investigaciones que permiten analizar y comprender el papel de la enseñanza en la educación social de los jóvenes. Las más importantes se han hecho en relación con la enseñanza de la historia.

En el análisis de las respuestas dadas por los jóvenes franceses en la macroencuesta citada anteriormente, Tutiaux-Guillon y Mousseau (1998), señalan:

"L'image de l'enseignement de l'histoire que choisissent les élèves est assez fidèle au fonctionnement de la classe. Ils affirment prendre plaisir à ces cours, qu'il s'agisse d'écouter le professeur ou de travailler sur documents, et faire confiance à l'enseignant et aux sources historiques. Les seueles discordances viennent d'élèves en situation difficile, en ZEP ou en classes technologiques. Ailleurs, l'adhésion est la règle. L'histoire récente et l'histoire de France intéressent (même si l'histoire familiale intéresse bien davantage). Les aprentissages factuels sont assez efficaces, surtout si l'on compare à l'ensemble des élèves d'Europe. Les jeunes reprennent à leur compte una image positive de l'histoire, qui, au-delà de la classe, sert à comprendre le présent, voire à s'orienter pour l'avenir. Un large groupe d'adolescents assigne même à l'histoire un rôle dans leur formation personnelle. Voilà de quoi, en apparence, conforter les enseignants dans leur effort: les éleves semblent atteindre certains des buts proclamés de l'histoire scolaire.

Mais, dès que les questions dépassanet les formules convenues, cette belle image s'effrite. La maîtrise des connaissances factuelles -ce qui ne veut pas dire de la culture générale - n'est jamais corrélée avec telle ou telle attitude. Les adolescents dissocient manifestement leur vie future, sous le signe du bonheur individuel, et le devenir social, plutôt redoutable. (...)

Mais, la question reste posée du divorce entre l'histoire telle qu'on l'enseigne au quotidien, et l'histoire telle que ces jeunes la comprennent, entre l'ambition de l'histoire scolaire à développer des identités collectives, à aider à devenir citoyen, de sa ville, de France ou d'Europe, et la faible place de l'his- 
toricité et de la politique dans les choix des adolescents. Car même si le questionnaire quêtait fortement les avis personnels, ceux-ci auraient pu faire émerger -comme dans d'autres pays - une adhésion aux valeurs collectives, un intérêt plus vigoureux pour la vie et l'histoire de la cité, et une moindre hétérogénéité des attitudes. Autrement dit encore, les savoirs factuels et les prises de position consensuelles valorisées par l'histoire scolaire ne sous-tendent guère la capacité à juger le monde et à penser l'avenirn (TutiauxGuillon/Mousseau, 1998: 212-123).

Esta valoración coincide bastante con la que realizó Lautier (1997) sobre la percepción que los jóvenes franceses tienen de la historia. Para esta investigadora, "les élèves ne dissocient pas les propriétés qui la caractérisent des fonctions qui lui sont associées. Pour eux, l'histoire est avant tout un moyen d'acquérir des connaissances $(92,5 \%)$; mais ils acceptent également, dans des proportions assez larges, les finalités associées à cette discipline: éveiller la curiosité $(75,5 \%)$, exercer la réflexion $(66,8 \%)$, déveelopper le sens de la tolérance (63\%). (...). Surtout, l'histoire enseignée paraît s'élargir, aux yeux des élèves, des fonctions purement scolaires à un niveau plus globalisant situé dans un vaet-vient entre le social et le privé». (pág. 49-50). Y concluye, más adelante, "Comme moyen de comprendre le monde, l'histoire ne prépare pas nécessairement à remplir son rôle dans la cité. La démarche se borne le plus souvent à une recherche identitaire directe, celle du Même plutôt que celle de l'Autre; les enjeux se limitent à la socialisation ou même à l'utilitarisme plus souvent qu'à la fonction civique» (pág. 55).

La capacidad de comprender el mundo y pensar el futuro tampoco la han aprendido los jóvenes italianos o, al menos, esto es lo que se desprende del comentario a las respuestas a una de las preguntas ${ }^{2}$ de la investigación dirigida por Baiesi y Guerra (1997) sobre las relaciones entre vida cotidiana y representación de la historia. Sus palabras son bastante concluyentes:

"(...) in poche parole, sembra riassumersi l'atteggiamento estremo di un'intera generazione, che si sente e si pensa out, fuori della storia, dichiaramente e consapevolmente proiettata in una dimensione che non è piu collettiva, dove il «noi» si conferma sempre più circoscritto alla ristretta del quotidiano: quella

2 La pregunta decía: «in questi ultimi cinquant'anni ci sono stati eventi storici in cui ti sei sentito coinvolto?" (pág. 110). 
della famiglia, ma anche quella della piccola "tribu", il gruppo rassicurante e stabile dei pari» (pág. 110-111).

No parece, sin embargo, que esta situación se dé entre los jóvenes españoles aunque existen pocas investigaciones al respecto. En las conclusiones de la macroencuesta ${ }^{3}$ "Youth and History", los responsables españoles Limón y Carretero (1997, p. A 342) afirman:

"After these results it can be said that our students' have a moderately positive view of history. They considered history as showing "the background of the present way of life and explaining today's problems", as "a means of mastering their life as part of historic changes" and as "a chance for myself to learn from failures and successes of othersm. They did not think that history was «something dead and gone, which has nothing to do with their present life». In comparison to the international sample, Spanish students showed a higher motivation in treating history".

A pesar de la valoración de Limón y Carretero, parece que tanto en España como en la mayor parte del mundo, la contribución de la enseñanza de las ciencias sociales y de la historia al proceso de socialización de los jóvenes es hoy prácticamente nula. Tal vez en el pasado tuvo un papel importante en la socialización de algunos sectores juveniles minoritarios (los que accedían a los estudios secundarios). Hoy, para recuperar el papel en el proceso de formación de los jóvenes hace falta uun insegnamento (...) attento al presente non meno che al passato, capace di superare una dimensione puramente antiquariae di favorire negli studenti l'acquisizione della capacità di orientarsi, (...), ossia essere in grado di muoversi consapevolmente in quell'orizzonte dai contorni incerti di cui si è detto ed insieme di esercitare la facoltà del guidizio e conquistare una propia capacità di scelta» (Baiesi/Guerra, 122).

\section{Ideas para seguir discutiendo...}

Quiero concluir estas reflexiones con tres sugerencias para resituar el debate de la enseñanza de los contenidos sociales e históricos en el terreno

3 Esta encuesta la contestaron un total de 991 jóvenes españoles de unos 15 años de la comunidad de Madrid. 
educativo. En primer lugar, es necesario potenciar mucho más la investigación en didáctica de la historia y de las ciencias sociales, y dar a conocer aquellas experiencias innovadoras que se están realizando en las aulas. Hay que ir más allá de los informes retóricos y de las opiniones interesadas y comprobar qué ocurre en las aulas. ¿Existen experiencias de enseñanza positivas?, ¿en qué se basan? Se debería poder comprobar, por ejemplo, si es cierta en España la afirmación de Whelan (1997) de que la investigación sobre la enseñanza de la historia en Estados Unidos y en el mundo anglosajón demuestra que los estudiantes aprenden mejor cuando son activos en su aprendizaje y no pasivos, creativos y no meramente receptivos, y cuando se les propone ejercer su juicio crítico y no simplemente memorizar información factual. La administración educativa, todas las administraciones educativas, deberían invertir más en investigación para poder tomar medidas adecuadas a lo que realmente ocurre en las aulas.

En segundo lugar, no se puede hablar de mejorar la enserianza sin repensar la formación del profesorado. En un mundo cada vez más complejo, más dominado por las tecnologías de la información y de la comunicación, hay que preparar al profesorado de una manera distinta a como se le ha preparado hasta hoy. Para que nuestros alumnos aprendan historia y ciencias sociales hay que enseñarles historia y ciencias sociales. Para enseñar historia y ciencias sociales se ha de saber historia y ciencias sociales, sin duda, pero también hay que saber enseñarlas. Y de la misma manera que se enseña a los profesores historia y ciencias sociales es necesario enseñarles a enseñarlas.

Y, finalmente, hay que afrontar los retos del presente con una clara visión de futuro. Y esta visión pasa, en mi opinión, por una enseñanza de la historia y de las ciencias sociales dirigida a la formación democrática de nuestro alumnado, de un alumnado que ha de poder utilizar los conocimientos históricos y sociales para interpretar y participar en su mundo como ciudadano crítico, con opinión propia, y con clara conciencia de que el futuro le deparará muchos cambios. Y uno de estos cambios será, sin duda, el de la existencia de una realidad cada vez más global, diversa y plural ante la cual no hay "historias ni humanidades comunes" que valgan sino tratan de los problemas comunes de los hombres y de las mujeres, y de la manera como en el pasado los solucionaron y como los solucionan en el presente. Si no tratan, en definitiva, de la diversidad cultural de ayer, hoy y mañana. 


\section{Bibliografía}

ANGVIK, M./Von BoRRIES, B. (1997): Youth and History. A comparative european survey on historical consciousness and political attitudes among adolescents. Vol. A. Description. Hamburg. Edition Jörber-Stiftung.

ARKELL, T. (1986): "History's role in the school curriculum». J. Education Policy, vol. $3, \mathrm{n}^{\circ} 1,23-38$.

BAIESI, N./GueRRA, E. (a cura di): Interpreti del loro tempo. Ragazze tra scena quotidiana e rappresentazione della storia. Bologna. CLUEB.

BruSA, A. (1999): “Il nuovo curriculo di storia». Ministero Pubblica Istruzione. Direzione Generale Istruzione Profesionale: ...»non e più la stessa storia!». Modena, 65-78.

CUESTA, R. (1999): «La educación histórica del deseo. La didáctica de la crítica y el futuro del viaje a Fedicaria». Con-Ciencia Social. Anuario de Didáctica de la geografia, la historia y otras ciencias sociales, n. ${ }^{\circ} 3,70-97$.

CUESTA, R. (del grupo Cronos) (coord.) (1999): "Editorial». Con-Ciencia Social. Anuario de Didactica de la geografia, la bistoria y otras ciencias sociales, $\mathrm{n}^{\circ}$ 3, 7-10.

DomenaCH, J.-M. (1989): Ce quil faut enseigner. Pour un nouvel enseignement genéral dans le secondaire. Paris. Éditions du Seuil.

Evans, R. W. (1997): “Teaching Social Issues». WaYne Ross, E. (ed.): The Social Studies Curriculum. Purposes, Problems, and Possibilities. Satate University of New York Press, Albany, 197-212.

Gimeno, P. (1999): «La Teoría Crítica de Habermas y la educación: hacia una didáctica crítico-comunicativa". Con-Ciencia Social. Anuario de Didáctica de la geografia, la historia y otras ciencias sociales, n. ${ }^{\circ} 3,13-41$.

Goodson, I. F. (1995): Historia del curriculum. Barcelona. Pomares.

Hargreaves, A. (1998): Profesorado, cultura y postmodernidad (Cambian los tiempos, cambia el profesorado). Madrid, Morata.

Johnson, T./Avery, P. G. (1999): "The Power of the Press: A Content and Discourse Analysis of the United States History Standards as Presented in Selected Newspaper». Theory ans Research in Social Education, vol. 27, n. ${ }^{\circ}$, 447-471.

KHOLI, W. (2000): "Teaching in the Danger Zone. Democracy and Difference". HURST, D.W./WAYNE ROSS, E. (ed.) (2000): Democratic Social Education. Social Studies for Social Change. New York/London. Falmer Press, 23-42. 
LAUTIER, N. (1997): A la rencontre de l'histoire. Villeneuve d'Ascq. Presses Universitaires du Septentrion.

LEEUW-ROORD, J. van der (ed.) (1998): The State of History Education in Europe: challenges and implications of the "youth and history" survey. Hamburg: KörberStiftung.

PAGES, J. (1991): "Al término de una etapa. Una opinión sobre la reforma del currículum de Ciencias Sociales, Geografia e Historian. Cuadernos de Pedagogia n. ${ }^{\circ} 197$, 70-73.

PAGES, J. (1998 $)$ : "Contenidos y formación del profesorado. La historia a debate». Cuadernos de Pedagogta n. ${ }^{\circ}$ 268, 82-84.

PAGÉs, J. (1998b): «La reforma de la enseñanza de las humanidades. El punto de vista de la Asociación Universitaria del Profesorado de Didáctica de las Ciencias Socialesm. Iber. Didáctica de las Ciencias Sociales, Geografia e Historia n. ${ }^{\circ} 16,83-$ 86.

PAGES, J. (1999): "Análisis crítico de las historias que se cuentan». MAYORAL, V. et al: Nacionalidad, historia y educacion. Madrid. Fundación Cives, 41-61.

PraTs, J. (2000): «Disciplinas e interdisciplinariedad: el espacio relacional y polivalente de los contenidos de la didáctica de las ciencias sociales». Iber. Didáctica de las Ciencias Sociales, Geografia e Historia n. ${ }^{\circ}$ 24, 7-17.

RozADA, J. M. (1999): "Ideas y dudas sobre una enseñanza crítica de las ciencias sociales en la escuela comprensivam. Con-Ciencia Social. Anuario de Didáctica de la geografia, la historia $y$ otras ciencias sociales, $n .^{\circ} 3,42-69$.

SAXE, D. W. (1997): "The Unique Mission of the Social Studies". WAYNE Ross, E. (ed.): The Social Studies Curriculum. Purposes, Problems, and Possibilities. Satate University of New York Press, Albany, 39-55.

STANLEY, W. B./NELSON, J. L. (1994): "The foundations of social education in historical context". MARTUSEWICZ, R./REYNOLDS, W. (eds.): Insidelout: Contemporany critical perspectives in education. New York. St. Martin" press, 266-284.

TUtAaux-guillon, N./MOUSSEAU, M.-J. (1998): Les jeunes et l'histoire. Identités, valeurs, conscience historique. Enquête européenne "Youth and history». Paris. Institut National de Recherche Pédagogique.

Wayne Ross, E. (1997): “The Struggle for the Social Studies Curriculum». WAYNE Ross, E. (ed.): The Social Studies Curriculum. Purposes, Problems, and Possibilities. Satate University of New York Press, Albany, 3-19. 
WaYNe Ross, E. (2000): "Redrawing the Lines. The Case against Traditional Social Studies Instruction". HURST, D.W./Ross, E.W. (ed.) (2000): Democratic Social Education. Social Studies for Social Change. New York/London. Falmer Press, 4363.

Whelan, M. (1997): "History as the Core of Social Studies Education". WAYNE Ross, E. (ed.): The Social Studies Curriculum. Purposes, Problems, and Possibilities. Satate University of New York Press, Albany, 21-37. 